\title{
Rage in Conjunction with the Machine
}

\author{
Mark Cartwright \\ Center for Computer Research in \\ Music and Acoustics (CCRMA) \\ Department of Music, Stanford \\ University \\ Stanford, CA 94305 \\ $+18473080038$ \\ mcartwri@ccrma.stanford.edu
}

\author{
Matt Jones \\ Department of Art \& Art History, \\ Stanford University \\ Stanford, CA 94305 \\ $+15052288987$ \\ mattjones@ojdingo.com \\ www.ojdingo.com
}

\author{
Hiroko Terasawa \\ Center for Computer Research in \\ Music and Acoustics (CCRMA) \\ Department of Music, Stanford \\ University \\ Stanford, CA 94305 \\ hiroko@ccrma.stanford.edu
}

\begin{abstract}
This report presents the design and construction of Rage in Conjunction with the Machine, a simple but novel pairing of musical interface and sound sculpture. The authors discuss the design and creation of this instrument, focusing on the unique aspects of it, including the use of physical systems, large gestural input, scale, and the electronic coupling of a physical input to a physical output.
\end{abstract}

\section{Keywords}

Audience participation, new musical instrument, musical instrument design, mapping, physical systems, sound scultpure, inflatable, instrument size

\section{INTRODUCTION}

While musical sound in and of itself is capable of conveying a wide range of expressive and emotional content, the visual component in a live or video-recorded musical performance can effect the expressive impact. In designing new performance interfaces, the mapping of physical interaction and resultant musical gesture often becomes obscured, leaving the audience to wonder why, for example, a particularly bold physical motion resulted in such a timid sound or vice versa. In this paper we explore the limitations of the common tripartite organization of musical instrument, human interaction with that instrument, and the auditory result of that interaction. Specifically, we describe an extension of these limitations in the context of an interactive performance system / installation consisting of two sculptures one comprising an interactive interface, and the other a sound generator. We discuss the implications of segregating the direct human manipulation of audio control from the processing and sound generation, as well as issues of size and dimension vis a vis retaining sufficient gestural control with the performer while providing a visual sense of expressive gesture to the audience.

Permission to make digital or hard copies of all or part of this work for personal or classroom use is granted without fee provided that copies are not made or distributed for profit or commercial advantage and that copies bear this notice and the full citation on the first page. To copy otherwise, or republish, to post on servers or to redistribute to lists, requires prior specific permission and/or a fee.

NIME07, June 7-9, 2007, New York, NY

Copyright remains with the author(s).
Rage in Conjugation with the Machine was the result of the collaboration of a musician, Mark Cartwright, and a sculptor, Matt Jones, to create a new musical instrument for audience interaction. The idea: give the audience a large, wireless, inflatable joystick to control a sound sculpture on stage during a musical performance. The creators hoped that because of the choice of controller and sound generator and their lucid mapping, novice users would engage in aggressive yet playful physical interaction with the instrument. This would ultimately end in the destruction of the sound generator and yield beautiful, cacophonous sonic results in the process.

While the concept behind a 5 ' joystick controlling the movement of a metal rod in a sea of percussion is rather simple, the unique choices of controller and sound generator provide solutions to what the authors consider common problems of NIMEs in a performance environment.

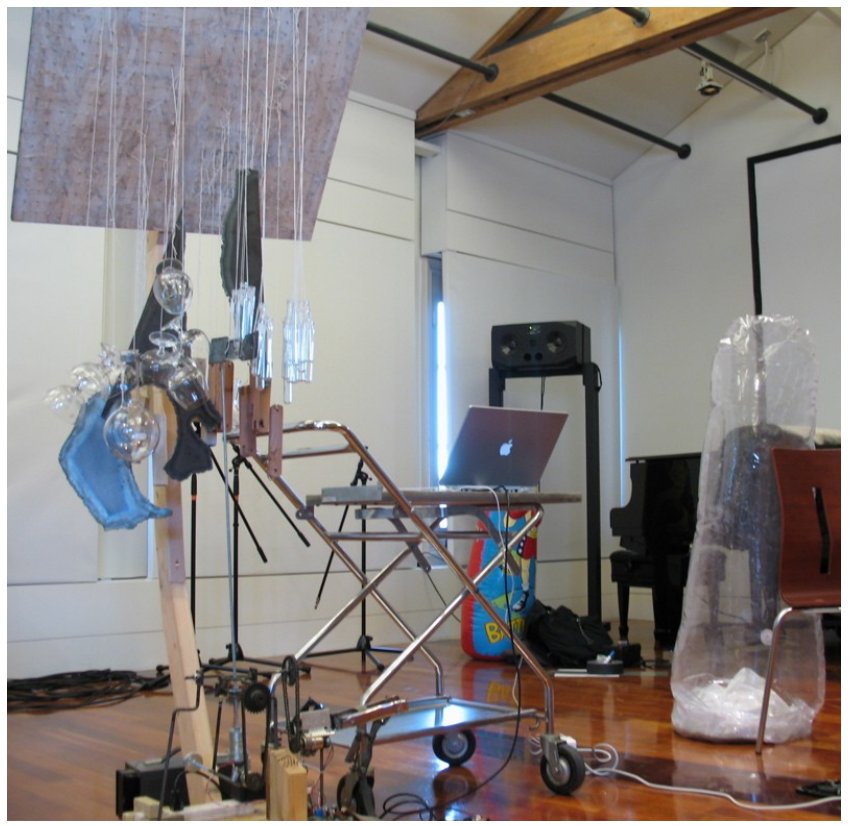

Figure 1. Rage in Conjunction with the Machine 


\section{MOTIVATIONS}

Initially Mark Cartwright's goal was to create an instrument to allow his audience to participate musically with him during performance. At his shows there is often a high energy in the audience that is displayed through dancing, yelling, bobbing of heads, etc. What if this energy could be used for making music? What kind of instrument would facilitate that sort of interaction?

In answering these questions, other questions arose. Traditional instruments have physical systems as inputs and outputs because these dynamic systems are often required for the control and generation of their sound. However, because of the use of electricity in NIMEs and the decoupling of controller and sound generator [1], most new interfaces do not have physical systems at inputs or outputs. What if an instrument had a physical system at the input and output, but they were coupled electronically?

\section{PHYSICAL INPUT}

The idea of "expressivity" is often brought up in the realm of NIME, and its definition is rather elusive. These authors define expressivity as the projection of one's personality into his/her actions. One can do this adroitly, eloquently, or elegantly, but expressivity is not dependent on these attributes. One of the easiest ways to project one's personality into his/her actions is to display it through bodily motion. It is not guaranteed that these actions will translate into musical expressivity, but a performance is more than just the sound arriving at the listeners' ears.

Large gestural motions are not ideal in all musical situations, especially those that require precise repeatability and/or prolonged virtuosic performance. However, for the potential novice users in an audience, a corporal approach to expressivity that requires little musical background seems ideal.

This type of interaction is created with Rage in Conjunction with the Machine by using a large bop bag as a joystick. A bop bag is a tall (usually a little less than the height of the user), upright, cylindrical inflatable that is weighted at the bottom so that when the top end is displaced, it returns to a zero position. A bop bag is traditionally used as a punching bag by both children and adults for exercise, play, and therapy. It therefore inherently elicits a somatic interaction with the user that can range from play to aggression. For most users, little, if any, explanation is required to explain the operation of this controller.

In addition to prompting large gestural motions from the user, the bop bag itself is an interesting system; because of its weighted bottom, it has physical dynamics of its own that impose themselves on the input of the instrument. While a simple system, the hope is that the addition of such subtle complexities will create a more engaging instrument.

\section{PHYSICAL OUTPUT}

NIMEs are often described in two parts: the controller and the sound generator [1]. Given this dichotomy, the authors propose that musical instruments can be categorized by their type of controller (virtual or physical), sound generator (virtual or physical), and the coupling between the two (virtual or physical). This yields 8 combinations (2 of which are redundant).

The model for type 3 instruments has been used in other fields of HCI, notably telerobotics [3], but the authors are unaware of any examples of this in musical instruments except for the instrument described in this paper.

The usefulness of this model of course depends on the goals of the instrument, but there are many benefits to NIMEs beyond just the control of electronic synthesis, and this model in particular addres ses many issues that commonly arise in NIMEs. For instance, the mapping of an instrument is not always transparent for a first time user or an observer [4]. While it depends on the complexity of the system, mapping to a physical output can potentially be clearer to the user and the observer since they can actually see the sound generator and how its actions correspond to its input; whereas, a virtual/electronic sound generator is often a "black box" which can only be deciphered with the help of aural clues. This mode of output inherently has complex sonic properties and physical dynamics that add aesthetic appeal like that of acoustic instruments. Unlike acoustic instruments though, an instrument of type 3 still has the control flexibility of an electronic instrument.

Since Rage in Conjunction with the Machine is meant for audience interaction, the lucidity of the mapping is an especially valuable characteristic, but the designers did not want to sacrifice sonic complexity for ease of use. Therefore, they chose a type 3 instrument model.

The physical sound generator, the sound sculpture, took the form of a tree of hanging metallic, glass, and wooden objects. At the base of this structure is a mechanized metal rod that can flail around this sea of percussion in response to the gestural input of the bop bag.

Table 1. Categorization of musical inst ruments by input, output, and coupling

\begin{tabular}{|l|l|l|l|l|}
\hline Type & Input & Coupling & Output & Example \\
\hline 1 & Physical & Physical & Physical & $\begin{array}{l}\text { Traditional Acoustic } \\
\text { Instruments/Sound } \\
\text { Sculptures }\end{array}$ \\
\hline 2 & Physical & Physical & Virtual & = TYPE 4 \\
\hline 3 & Physical & Virtual & Physical & $\begin{array}{l}\text { Rage In } \\
\text { Conjunction with } \\
\text { the Machine }\end{array}$ \\
\hline 4 & Physical & Virtual & Virtual & $\begin{array}{l}\text { Most electronic } \\
\text { instruments/NIME }\end{array}$ \\
\hline 5 & Virtual & Physical & Physical & == TYPE 7 \\
\hline 6 & Virtual & Physical & Virtual & None? \\
\hline 7 & Virtual & Virtual & Physical & LemurBots [2] \\
\hline 8 & Virtual & Virtual & Virtual & $\begin{array}{l}\text { Sequenced/Algorith } \\
\text { mically controlled } \\
\text { soft-synths }\end{array}$ \\
\hline
\end{tabular}




\section{SIZE AS SCULPTURAL ELEMENT IN NIME DESIGN}

The creators' choice of a 5 ' joystick raises another concern in NIME design. In this work, what is often a handheld, interactive object is enlarged and presented to the user in order to invite a different kind of interaction.

Size is an important aspect of sculptures when defining the perspective of the audience. Changing the mode of perception by scaling has been a concern in modern sculptures: Ron Mueck enlarged a lady lying in a bed to $6.5 \mathrm{~m}$ in his work "In Bed. [5]" Tony Oursler projected the movement of human eyes onto numerous huge white balls in "Mirror Maze (Dead Eyes Live.) [6]" Jeff Koons constructed a three meter tall "Balloon Dog (magenta)" [7] with high-chromium stainles. All of these works set the audience's perspective by changing the size of the objects.

Scaling also affects human interaction with objects. A good example is Isamu Noguchi's Moerenuma Park project [8], which includes play sculptures for children. Many of the sculptures actually have shapes that are reminiscent of toys such as small wood blocks, but because of their enlarged size, children play with the sculpture in a very athletic manner.

Human computer interaction with visual display is also affected by size. Guiard et al. [9] investigated miniaturized and enlarged interfaces and showed the improved efficiency of interaction in multi-scale navigation.

This demonstrates that scaling invites diversity in the way interaction occurs. In traditional musical instrument design, size was the most important factor in defining the pitch of the instrument. In contrast, this work, Rage in Conjunction with the Machine, suggests that with the aid of computers we are now liberated from scale constraints in instrument design and are able to choose the size appropriate for bodily expressions; a sound sculpture could exist beyond the limitations of size and material for sound production. As discussed by Gurevich and Treviño[10], the visual (or sculptural) element is one of the emerging factors in the diversity of aesthetic considerations in contemporary music and extends musical expressivity not merely with sounds but also with bodily motions.

\section{CONSTRUCTION}

\section{Bop Bag}

The control data, the tilt of the bop bag, was obtained by using a 3 -axis accelerometer. Since one of the goals of the instrument was to create an interface for audience interaction, the controller had to be wireless. This was necessary so that it can move freely around the audience. Given these requirements, the perfect candidate for the sensing implementation was the SparkFun WiTilt 2.5 [11].

In order to make the bop bag visible in a dark music performance environment, two high-powered LEDs (Lumileds [12]) were needed to illuminate the translucent inflatable. These, along with the accelerometer board, were protected by embedding them near the base of the bop bag. To accomplish this, the bop bag had two adjoined inflatable bladders: a small, half beach ball sized one attached to the weighted base, and the large, main bladder. The boards were sandwiched between the two, and were accessible through an opening on the base of the side that could be fastened shut with snaps.

The structure of the bop bag was created out of clear poly-vinyl chloride (PVC), and was adhered together with vinyl cement. Like most bop bags, the bottom was weighted with sand.

\section{Coupling}

The bop bag and the sculpture were electronically coupled through a PowerBook G4 and an Atmel AVR32 microcontroller. The tilt information from the accelerometer was transmitted via Bluetooth to the PowerBook, which processed the input data in MaxMSP and sent the output control data via serial bus to the microcontroller. The microcontroller parsed out the serial data and controlled the two motors (x,y) with a AVR Motor Controller board.

\section{Sculpture}

The sculptural machine was built to resemble an upside-down pendulum, standing about six feet tall. The pendulum (1/4" steel rod) was motorized to crash into a chandelier of hanging material, selected for its percussive properties (primarily test tubes, brandy snifters, and steel plates). The pendulum had two axes of motion, driven by two high-torque DC motors geared down to swing the two axes of motion. To reduce strain on the motors, all axes use bearings and are carefully counterbalanced, using fender washers mounted on threaded rod. But recognizing that the machine was to be built with common metal and wood tools, we opted for a less precise, higher tolerance approach for the design of the machine. The reduced precision hindered calculated playing of the instrument, but the simpler mechanism allowed for the unique personality of the machine to emerge, much like the odd quirks of an old car.

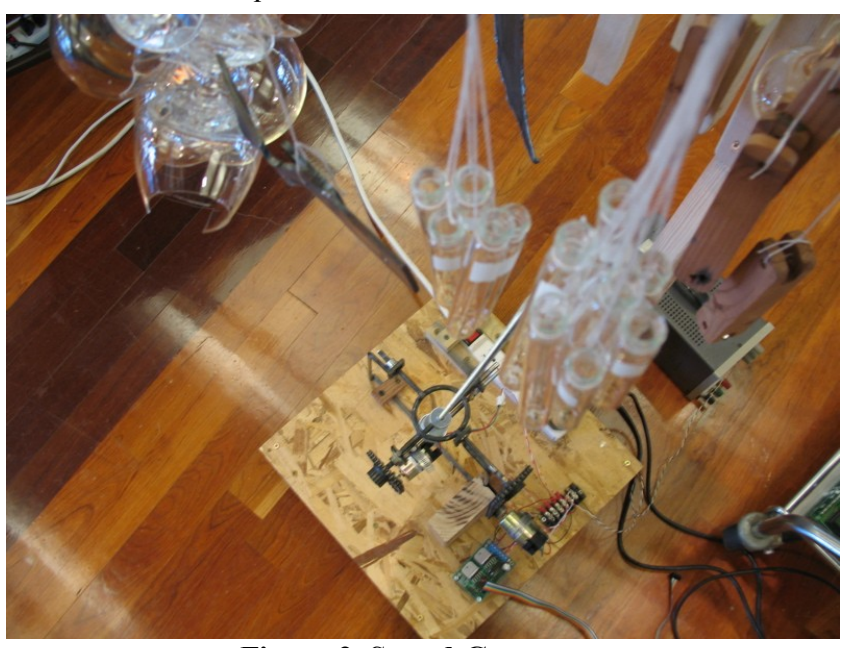

Figure 2. Sound Generator

\section{PERFORMANCE}

Due to construction methods of the bop bag and the difficulties of building robust DIY inflatables, Rage in Conjunction with the Machine did not last its first demonstration. The seams of the bop bag gave out under the relentless aggression of the user. In addition to this design flaw, the motors used were a bit 
underpowered. Although first seen as a problem, the weak output of the motors gave the instrument an interesting personality that seemed to have some of the characteristics of a resonant system. For instance, in order to get the mechanized arm to obtain maximum displacement, the user would need to rock the bop bag back and forth at a certain rate. Moving it faster than this yielded virtually no effect, and moving it slower than this created more of a slow, mimicking response but with less displacement.

These type of responses in two dimensions do not yield a simple 1:1 mapping as the initial coupling would suggest, but rather a complex control relationship that forces the user to address the needs of the machine and meet it halfway, possibly changing the user's attack strategy on the bop bag; yet, because both the controller and sound generator were visible physical systems, the mapping was not obscure. Users and observers were able to understand the dynamics of the system based on their knowledge of physical systems obtain throughout their life. A response like this also requires the user to become even more somatically engaged in the performance, creating a fun, visually exciting, and "expressive" performance for all involved.

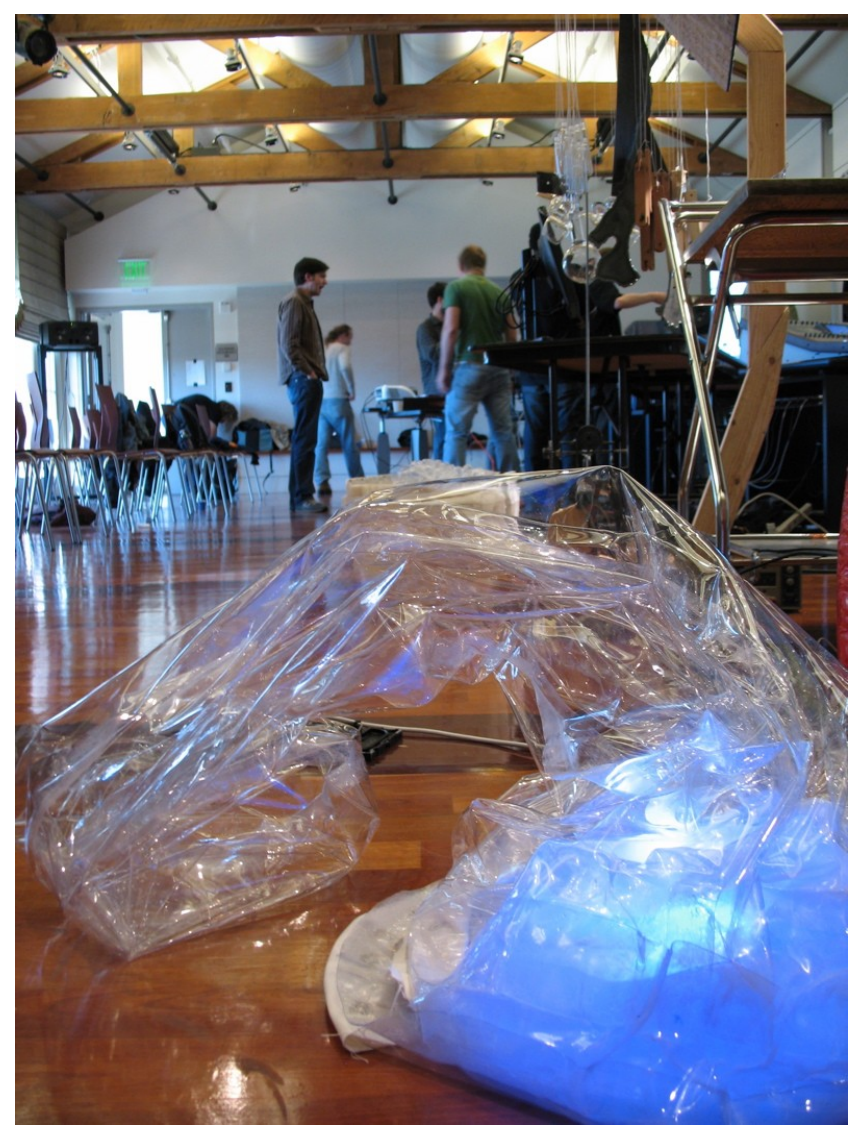

Figure 3. The death of the bop bag

\section{CONCLUSIONS}

In this paper, the authors discussed the categorization of musical instruments in terms of input, output and coupling formats, which expands the view and possibility in the NIME design. In addition, sculptural elements are discussed in terms of the perception of scale and its effect on interaction in the context of aesthetics of new music composition.

\section{ACKNOWLEDGEMENTS}

The authors would like to thank Jonathan Berger, Michael Gurevich and Bill Verplank for their help and support in getting this project and paper finished.

\section{REFERENCES}

[1] J. Chadabe. The Limitations of Mapping as a Structural Descriptive in Electronic Instruments. In Proceedings, Conference on New Interfaces for Musical Expression (NIME-02), 2002.

http: //hct .ece.ubc.ca/nime/2002/proce edi ngs/paper/chadabe_keynote.pdf.

[2] E. Singer, J. Fedderson, C. Redmon, and B. Bowen. LEMUR's Musical Robots. In Proceedings, Conference on New Interfaces for Musical Expression (NIME04), 2004. http: / / hct .ece.ubc. ca/nime/2 004 /NIME0 4/p aper/NIME0 4_3D01.pdf.

[3] C. Melchiorri. Robotic Telemanipulation: An Introduction. http: //isa .umh.es/vr2/euron0 6/doc/Mel chi orri/Benidorm_Telemanip_Part_1.pdf.

[4] S. Fels, A. Gadd, and A. Mulder, "Mapping transparency through metaphor: toward more expressive musical instrument s," Organized

[5] R. Mueck. "In Bed" Mixed Media Sculpture, 2005. Collection of the foundation Cartier for Arts.

[6] T. Oursler, "Mirror Maze (Dead Eyes Live)" Mixed Media, Video, Projector. 2003. Collection of the foundation Cartier for Arts.

[7] J. Koons. "Balloon Dog (Magenta)" Stainless steel. 19942000. Francois Pinault Collection.

[8] Noguchi, I. "Moer enuma Park.” Play sculptures. 19882005.

[9] Y. Guiard, et al. "View size and pointing difficulty in multiscale navigation." Proceedings of Advanced Visual Interfaces (AVI). New York: ACM Press., pp. 117-124. (2004.)

[10] M. Gurevich and J. Treviño. "Expression and Its Discontents: Toward an Ecology of Musical Creation." Submitted to NIME 2007.

[11] Spark Fun. http: / /www . sparkfun .com/ .

[12] Lumileds. http: / /www . lumileds .com. . 